\title{
Factors Associated with Health Extension Service Utilization in Dera District, Oromia, Ethiopia: A Multi Level Analysis
}

\author{
Abate Tadesse Zeleke ${ }^{1,}$, , Dejen Tesfaw Molla ${ }^{2}$, Nega Assefa Kassa ${ }^{3}$ \\ ${ }^{1}$ Department of Statistics, Madda Walabu University, Bale Robe, Ethiopia \\ ${ }^{2}$ Department of Statistics, Addis Ababa University, Addis Ababa, Ethiopia \\ ${ }^{3}$ Department of Public Health, Haramaya University, Haramaya, Ethiopia \\ Email address: \\ sifabate10@gmail.com (A. T. Zeleke),dejentm@gmail.com (D. T. Molla),negaassefa@yahoo.com (N. A. Kassa) \\ *Corresponding author
}

\section{To cite this article:}

Abate Tadesse Zeleke, Dejen Tesfaw Molla, Nega Assefa Kassa. Factors Associated with Health Extension Service Utilization in Dera District, Oromia, Ethiopia: A Multi Level Analysis. American Journal of Theoretical and Applied Statistics. Vol. 8, No. 3, 2019 , pp. 85-93. doi: $10.11648 /$ j.ajtas.20190803.11

Received: April 4, 2019; Accepted: May 20, 2019; Published: July 30, 2019

\begin{abstract}
Introduction: Health Extension Program is implemented in Ethiopia since 2004 as a flagship of preventive, promotive, and basic curative services for poor and rural community, especially for children and mother. The purpose of this study is to examine the determinants and variations in health extension service utilization in Dera district, Oromia, Ethiopia. Methodology: Three-stage cluster sampling was used to select a random sample of 534 households from 10 kebeles of the district. The study used both descriptive and inferential statistics to identify the determinants of Health extension service utilization and variations. Result: The descriptive result revealed that out of 534 respondents, 223 (41.8\%) of them utilized and 311 (58.2) of them not utilized health extension service given at the health post and outreach level. The random intercept binary logistic regression analysis identified that mother's age, mother educational level, mothers' employment status, knowledge on Health Extension Program, being model in Health Extension Program implementation, frequency of home visited by Health Extension Workers, number of students in a family and perception about conduct of Health Extension Workers were significant determinants of Health Extension Program utilization. Conclusion: Comparison between multilevel models has done and random intercept binary logistic model was found to have a better fit for utilization of health extension program and the district health office has to work to reduce the variance and factors that affect Health Extension Program utilization for each kebeles of the district.
\end{abstract}

Keywords: Dera, Health Extension Program, Multilevel Logistic and Utilization

\section{Introduction}

The Ethiopian Federal Ministry of Health launched the Health Extension Program (HEP), a flagship program of health of the Government of Ethiopia, was launched by the Federal Ministry of Health in 2003 with the goal of improving health outcomes in Ethiopia by targeting households and communities and implemented in 2004 [1]. Health extension workers (HEWs) promote health at the kebele (village) level by implementing 16 health service packages [2].

The HEP delivers a package of basic and essential promotive, preventive, and curative health services targeting households in a community, based on the principles of primary health care to improve families' health status with the active participation of both households and the community [1]. Hence, health extension workers are posted to rural communities across Ethiopia, where they provide better and more equitable access to health services for the poor, women, and children in a sustainable manner [3, 4].

The health extension program (HEP) is an innovative community level component of the Essential Health Services Package. The primary purpose of the HEP is to improve access and utilization of preventive, promotive, and basic 
curative services for poor and rural community, especially for children and mother in the country by creating opportunities to enable households to exercise, a health Practice and living healthy through comprehensive, interrelated, economically and technically feasible health interventions $[4,5]$.

Each kebele has a health post that serves 5,000 people and functions as an operational center for a health extension worker. Five health posts and a health center work in collaboration and for the Primary Health Care Unit (PHCU) that serves 25,000 people. The health post is under the supervision of the district health office and the kebele administration and receives technical and practical support from the nearby health center $[1,5]$. However, all HEP services, which are given at the health post and outreach by health extension workers are not properly utilized in the country as well as in the district [6]. In addition, in the studyarea (Dera district) to the knowledge of researchers, no quantitative study has been carried out to quantify how the factors influence utilization of HEP. Hence, this study seeks to discover how demographic, socioeconomic and HEWs related variables influence community's utilization of HEP and statistically quantify variations in HEP utilization.

\section{Research Methodology}

\subsection{Descriptions of Study Area and Population}

Dera is one of the 18 districts of the North Shewa Administrative Zone, which is located in Oromia Regional State of Ethiopia. It is $213 \mathrm{~km}$ far from Addis Ababa, the capital city of Ethiopia and bordered on the South by the Jamma River, which separates it from Hidabu Abote and Wara Jarso, on the West, North and East by the Amhara Region. The district is located between $12^{\circ} 92^{\prime}-13^{\circ} 12^{\prime} \mathrm{N}$ latitude and $34^{\circ} 40^{\prime}-35^{\circ} 80^{\prime} \mathrm{E}$ longitude and elevation from $1798 \mathrm{~m}$ to $2118 \mathrm{~m}$ above sea level. The administrative center of Dera is Gundo Meskel [7].

The district has at total populations of 223,218 among these 115,442 are male and 117,778 are female [8]. The district has seven health centers and 34-health posts in 33 rural kebeles and 1 semi urban kebeles. These 34 health posts were nesting in seven health centers [6].

\subsection{Study Design}

A community based cross-section study was conducted on randomly selected women from February 10-30, 2016, who fulfill the two inclusion criteria. (1) Household who was living more than one year at the study area (2) women who aged 15-49 years old and has at least one child who aged less than five years old.

\subsection{Sampling Techniques}

The study employed multistage sampling technique to select the study units (women). At the first stage, 5 out of 7 health centers (Gundo Meskel, Tuti, Harbu Meskele, Cheka and Racho) were selected based on their geographical location. At the second stage, ten kebeles were selected using proportion to size of health centers. The ten sampled kebeles (clusters) are Dembi Birjie, Kabi Gololcha and Ada'a Melke from Gundo Meskel HC, Gebro and Keraba from Tuti HC, Becho Wajitu and Makefta Jiru from Harbu Meskele HC, Were Gebro and Weglo Mika'el from Cheka HC and Amuma Gendo from Racho HC. This is based on different studies [9, 10] suggested number of clusters for estimation and inference concerning multilevel regression. Lastly, at third stage women who satisfy the inclusion criteria were selected systematically.

\subsection{Sample Size Determination}

Using the previous research work proportion of the households who utilized HEP service $(p=0.39)$ [11], total target population size $\mathrm{N}=10290$ [6], significance level $\alpha=$ 0.05 , margin of error $d=0.05$ and $\operatorname{def}=1.5$ is a minimum default value of design effect for clustered data sets [12]. Then, the sample size computed as:

$$
\mathrm{n}=\frac{\text { no }}{1+\frac{\mathrm{no}}{\mathrm{N}}} * \operatorname{deff}
$$

Where: $-\mathrm{n}$ is the total sample size for clustered

$$
\begin{aligned}
& \mathrm{n}_{\mathrm{o}}=\frac{\left(\mathrm{z} \frac{\alpha}{2}\right)^{2} \mathrm{p}(1-\mathrm{p})}{\mathrm{d}^{2}}=\frac{(1.96)^{2} 0.39 * 0.61}{0.05^{2}}=365.56=366 \\
& n=\frac{366}{1+\frac{366}{10290}}=355.3=356 * 1.5=534
\end{aligned}
$$

Then, the overall sample size of 534 women was allocated to each kebele using proportion to size allocation as in the following Table 1.

Table 1. Proportional allocation of samples to kebele.

\begin{tabular}{llllll}
\hline Sampled kebele & Target HH size & Sampled HH per kebele & Sampled kebele & Target HH size & Sampled HH per kebele \\
\hline Dembi Birjie & 840 & 44 & Becho Wajitu & 732 & 38 \\
Kabi Gololcha & 1486 & 77 & Makefta Jiru & 1542 & 70 \\
Ada'a Melke & 1162 & 60 & Were Gebro & 746 & 903 \\
Gebro & 1265 & 66 & Weglo Mika'el & 83 \\
Keraba & 782 & 40 & Amuma Gendo & 830 \\
N total = 10290 & & & Sample total $=534$ & 43 \\
\hline
\end{tabular}


Response variable: is utilization of health extension service. If a women utilize above the $50 \%$ health extension packages, she is considered as utilizing health extension service and coded as Utilize $=0$, otherwise Not and coded as not utilize $=1$.

Independent variables: the independent variables of the study classified as individual and Kebele level variables. The variables such as Mother age, education level, employment status, distance to health post, frequency of home visited, being model in HEP, knowledge on HEP and perception of women about HEWs conduct classified as individual level where as mean year service of HEWs and kebele were considered as cluster (kebele) level variable.

\subsection{Methods of Data Analysis}

The statistical methods used in this study were descriptive statistics (barchart, percentages and mean) and inferential statistics (multilevel regression model).

\subsubsection{Multilevel Logistic Regression Model}

Multilevel logistic regression model used to predict a binary response variable from a set of independent variables [13]. This study examined the effect of individual and kebele level variables using a two-level multilevel modeling. During analysis, the characteristics of women (households) were taken as individual level (level 1), and kebeles were treated as level two and multilevel binary logistic regression model is used to model utilization of HEP.

(i) Heterogeneity of proportion

The basic data structure of the two-level logistic regression model is a collection of $\mathrm{M}$ groups (kebele units at level two) and within-group $\mathrm{j}(\mathrm{j}=1,2 \ldots \mathrm{M})$ a random sample of $\mathrm{n}_{\mathrm{j}}$ levelone units (women). The outcome variable, utilization of HEP is dichotomous. Yij denotes the outcome variable for women $i$ in Kebele $j\left(i=1,2 \ldots n_{j}, j=1,2 \ldots M\right)$. To test whether there are indeed systematic differences between the groups, the well-known chi-square test for contingency table can be used. The test statistic is:

$$
\chi^{2}=\Sigma\left(\frac{(O-E)^{2}}{E}\right)
$$

Where: - $\mathrm{O}$ is observed and $\mathrm{E}$ is the expected count in the cell of the contingency table.

This can be written as:

$$
\chi^{2}=\frac{\sum_{j=1}^{N} n_{j}\left(\hat{\pi}_{j}-\hat{\pi}\right)^{2}}{\hat{\pi}(1-\hat{\pi})}
$$

Where: $-\hat{\pi}_{j}=\frac{1}{n_{j}} \sum_{i=1}^{n_{j}} y_{i j}$ is the proportion that is not utilizing HEP service in kebele $\mathrm{j}$. and $\hat{\pi}=\frac{1}{n} \sum_{j=1}^{M} \sum_{i=1}^{n j} Y_{i j}$ is the overall proportion of women who not utilize HEP.

This statistic $\left(\chi^{2}\right.$ chi-square statistic) follows approximately central chi-square distribution with M-1 degrees of freedom [9].

(ii) The empty model

Empty model contains no explanatory variables at all that serves as a point of reference with which other models are compared. The empty two-level model for a dichotomous outcome variable refers to population of groups (level-2 units, kebele) and specifies the probability distribution for the group-dependent probabilities in equation $Y_{i j}=\pi_{j}+\varepsilon_{i j}$, without considering further explanatory variables. The empty level two logistic regression model is expressed by:

$$
\operatorname{logit}\left(\pi_{j}\right)=\beta_{0}+U_{0 j}
$$

Where:- $Y_{\mathrm{ij}}$ is the outcome variable for individual $\mathrm{i}$ ingroup $\mathrm{j}, \pi_{j}$ is the probability in-group $\mathrm{j}, \varepsilon_{i j}$ is individualdependent residual, $\beta_{0}$ the population average of the transformed probabilities and $U_{0 j}$ the random deviation from this average for group $\mathrm{j}$. This model does not include a separate parameter for the level-one residual variance of the dichotomous outcome variable. It follows directly from the success probability $\operatorname{Var}\left(\varepsilon_{i j}\right)=\pi_{j}\left(1-\pi_{j}\right)$. The residual $\varepsilon_{i j}$

$\varepsilon_{i j}$ are assumed to have mean zero and variance $\sigma_{\varepsilon}^{2}$ [9].

(iii) The random intercept model

The Random intercept model is used to model unobserved heterogeneity in the overall response

by introducing random effects. In the random intercept model the intercept is the only random effect, meaning that the groups differ with respect to the average value of the response variable, but the relation between explanatory and response variables cannot differ between groups. The random intercept model expresses the log odds, i.e. the logit of $P_{i j}$, as a sum of a linear function of the explanatory variables. That is, $\operatorname{logit}\left(P_{i j}\right)=\log \left[\frac{P_{i j}}{1-P_{i j}}\right]=\beta_{0 j}+\beta_{1} x_{1 i j}+\beta_{2} x_{2 i j}+\ldots+\beta_{k} x_{k i j}$

$$
=\beta_{0 j}+\sum_{h=1}^{k} \beta_{h} x_{h i j}, i=1,2, \ldots n_{j}, j=1,2, \ldots m
$$

Where: $\beta_{0 j}$ is assumed to vary randomly and is given by the sum of an average intercept $\beta_{0}$ and group-dependent deviations $U_{0 j}$ that is $\beta_{0 j}=\beta_{0}+U_{0 j}$ as a result: $\operatorname{logit}\left(P_{i j}\right)=\beta_{0}+\sum_{h=1}^{k} \beta_{h} x_{h i j}+U_{0 j}$

Where: $\beta_{0 j}+\sum_{h=1}^{k} \beta_{h} x_{h i j}$ is the fixed part of the model. The remaining $U_{0 j}$ is called the random or the stochastic part of the model. It is assumed that the residual $U_{0 j}$ is mutually independent and normally distributed with mean zero and 
variance $\sigma_{u}^{2}[9]$.

(iv) The random coefficient model

The random coefficients build up on the random intercept model by allowing the effects of individual level predictors to vary randomly across the region. In the random coefficient model both the intercepts and slopes are allowed to differ across the region. The multilevel random effect coefficients logistic regression model is based on linear models for the $\log$ odds that include random effects for groups (higher levels). Suppose that there are $\mathrm{k}$ level-one explanatory variables $X_{2}, \ldots, X_{k}$, and consider the model where all Xvariables have varying slopes and random intercept. That is

$$
\operatorname{logit}\left(P_{i j}\right)=\log \left[\frac{P_{i j}}{1-P_{i j}}\right]=\beta_{0 j}+\beta_{1 j} x_{1 j}+\beta_{2 j} x_{2 i j}+\ldots+\beta_{k j} x_{k i j}
$$

Where: $\beta_{0 j}=\beta_{0}+U_{0 j}$ and $\beta_{h j}=\beta_{h}+U_{h j}, \mathrm{~h}=1,2, \mathrm{k}$ as a result

$$
\operatorname{logit}\left(P_{i j}\right)=\log \left[\frac{P_{i j}}{1-P_{i j}}\right]=\beta_{0}+\sum_{h=1}^{k} \beta_{h j} x_{h i j}+U_{0 j}+\sum_{h=1}^{k} U_{1 j} X_{1 i j}
$$

Where: $\beta_{0}+\sum_{h=1}^{k} \beta_{h j} x_{h i j}$ is called the fixed part of the model and and the second part $U_{0 j}+\sum_{h=1}^{k} U_{1 j} X_{1 i j}$ is called the random part of the model. $U_{\mathrm{oj}}, \mathrm{U}_{\mathrm{ij}}, \ldots, \mathrm{U}_{\mathrm{kj}}$ are assumed independent between groups but may be correlated within group. The vector $\mathrm{U}_{\mathrm{oj}}, \mathrm{U}_{\mathrm{ij}, . .}, \mathrm{U}_{\mathrm{kj}}$ are independently distributed as a multivariate normal distribution with zero mean vector and variance-covariance matrix ' $\Omega$ [9].

\subsubsection{Parameter Estimation and Model Comparisons}

The most common methods for estimating multilevel logistic models are based on likelihood. Among the methods, the Marginal Quasi Likelihood or MQL and Penalized Quasi Likelihood or PQL are the two prevailing approximation procedures. Both MQL and PQL are based on Taylor series expansion to achieve the approximation. Based on the usage of the first and second term of the Taylor expansion, MQL and PQL are often known as first order MQL and secondorder MQL, first-order PQL and second order MQL respectively. After applying these quasi likelihood methods, the model is then estimated using iterative generalized least squares (IGLS) or reweighted IGLS (RIGLS). The most frequently used kind of approximation method used are based on a first-order or second-order Taylor series expansion of the link function [14]. Hence the study used second-order PQL.

Model comparison, and selection was done using Loglikelyhood difference and Bayesian information criteria (BIC) used in the analysis. A model with a lower BIC is preferred over a model with a larger BIC.

\section{Results and Discussions}

\subsection{Geographical Distribution of Health Extension Service Utilization}

A Majority of 311(58.2\%) of the respondents did not utilize Health extension service while $223(41.8 \%$ ) of them utilized of among the total sampled respondents. In addition to this, the study shows that the percentages of not utilizing HEP differ among the sampled kebeles of the district. The highest percentages of not utilizing HEP were observed in Dembi Birjie kebele (75.0\%) followed by Makefta Jiru (72.5\%) whereas the lowest percentages were observed in Were-Gebro were kebele (33.3\%) and followed by Keraba $(35.0 \%)$. (figure 1)

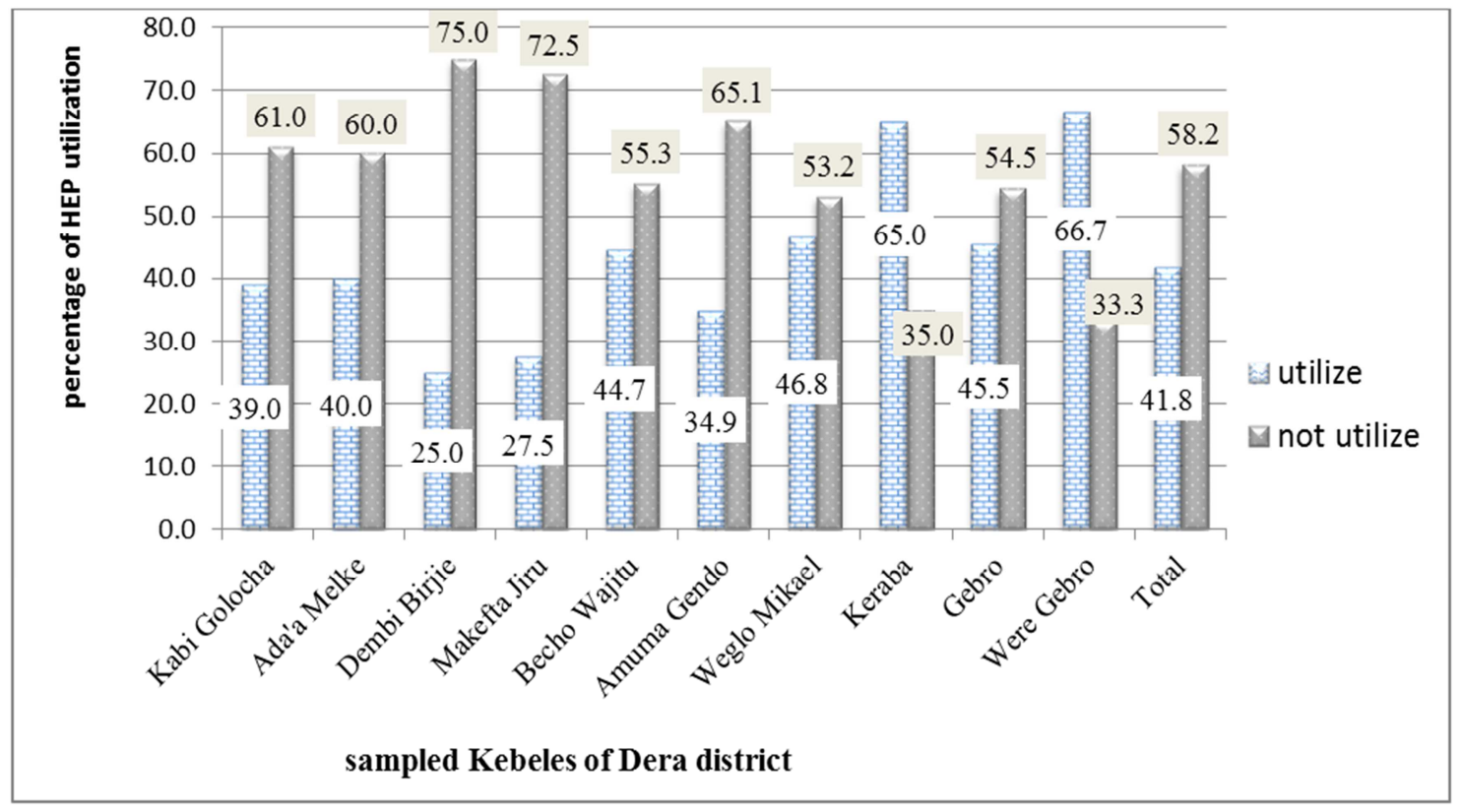

Figure 1. Geographical distribution of HEP Utilization in Dera district. 


\subsection{Some Demographic and Socioeconomic Character vs Utilizations of HEP}

Respondents' demographic and socioeconomic characteristics were analyzed based on their health extension program utilization. The highest percentages of not utilizing HEP $(64.0 \%$ and $57.3 \%)$ were observed in the age group 25 34 and 35-49, respectively, and the lowest percentage (35.8\%) was observed in the age group 15-24 (Table 2).

Based on the education level of the respondent, about $68.8 \%, 40.0 \%$ and $25.9 \%$ of the respondent who had no education, primary education, and secondary and above education did not utilize HEP, respectively. In addition to this, the study show based on husband education level about $62.3 \%, 59.5 \%$ and $46.8 \%$ of respondent whose husband had no education, primary education and secondary and above education did not utilize HEP, respectively.

Based on family size of the respondent, about $59.4 \%$ and $57.0 \%$ of the respondent who had less than and more than five family size did not utilize HEP, respectively. In addition to this, the study shows that about $69.8 \%, 64.2 \%, 41.9 \%$ and $41.2 \%$ of daily laborer, farmer, Housewife and Traders did not utilize HEP, respectively.

Table 2. Demographic and socioeconomic character vs utilizations of HEP.

\begin{tabular}{lllllll}
\hline \multirow{2}{*}{ Variables } & \multicolumn{7}{l}{ Utilization of HEP } & & \\
\cline { 2 - 6 } & Utilize & Percent & Not utilize & Percent & Total \\
\hline Mother age group & & & & & \\
$15-24$ & 43 & 64.2 & 24 & 35.8 & 67 \\
$\begin{array}{l}\text { Ab-34 } \\
\text { Above 35 }\end{array}$ & 104 & 36.0 & 185 & 64.0 & 289 \\
Education of wife & 76 & 42.7 & 102 & 57.3 & 178 \\
No education & 114 & 31.2 & 251 & 68.8 & 365 \\
Primary & 69 & 60.0 & 46 & 40.0 & 115 \\
Secondary and above & 40 & 74.1 & 14 & 25.9 & 54 \\
Education of husband & & & & & \\
No education & 135 & 40.5 & 198 & 59.5 & 333 \\
Primary & 46 & 37.7 & 76 & 62.3 & 122 \\
Secondary and above & 42 & 53.2 & 37 & 46.8 & 79 \\
Family size & & & & & \\
Less than or equal to5 & 115 & 40.6 & 168 & 59.4 & 283 \\
Greater than 5 & 108 & 43.0 & 143 & 57.0 & 251 \\
Mother's employment & & & & & \\
Farmer & 121 & 35.8 & 217 & 64.2 & 338 \\
Daily labor & 13 & 30.2 & 30 & 69.8 & 43 \\
Trade & 10 & 58.8 & 7 & 41.2 & 17 \\
Housewife & 79 & 58.1 & 57 & 41.9 & 136 \\
\hline
\end{tabular}

\subsection{Health Extension Related Character vs Utilization of HEP}

Based on the respondent graduation status of a model family, $70.2 \%$ and $37.9 \%$ of the respondent did not graduate and graduate as the model not utilize HEP, respectively. In addition to this, the study showed that majority $(71.9 \%$ of respondent who did not use Health post did not utilize HEP while $52.8 \%$ of those who use health post did not utilize HEP (Table 3).

Similarly, the study showed that about $77.0 \%, 65.5 \%$ and $44.4 \%$ of respondent who had bad, fair and good Attitude for
Health extension worker did not utilize HEP, respectively. Likewise, based on knowledge on HEP, about $78.6 \%$ and $46.8 \%$ of the respondent who had Unsatisfactory and satisfactory knowledge on HEP did not utilize HEP, respectively.

Based on respondents distance to health post about $63.4 \%$ and $54.8 \%$ of those who were far more than and less than 5 $\mathrm{km}$ from health post did not utilize HEP, respectively. Likewise, based on mean service year of HEWS at the health Post, about $57.5 \%$ and $62.0 \%$ of those who had HEWs served less than and more than 4year did not utilize HEP, respectively.

Based on the number times the home of the household visited in last 12 months by the HEWs, about 73.6\%, 46.9\%, $34.9 \%$ and $36.7 \%$ of the respondent whose home was Not visited, visited once, twice and three times by HEWs were did not utilize HEP, respectively.

Table 3. Health extension character vs utilizations of HEP.

\begin{tabular}{llllll}
\hline \multirow{2}{*}{ Variables } & \multicolumn{7}{l}{ Utilization of HEP } & & \\
\cline { 2 - 6 } & Utilize & Percent & Not utilize & Percent & Total \\
\hline Being Model family & & & & & \\
No & 100 & 29.8 & 238 & 70.2 & 336 \\
Yes & 123 & 62.1 & 75 & 37.9 & 198 \\
Use Health Post & & & & & \\
No & 43 & 28.1 & 110 & 71.9 & 153 \\
Yes & 180 & 47.2 & 201 & 52.8 & 381 \\
Altitude for HEWs & & & & & \\
Bad & 20 & 23.0 & 67 & 77.0 & 87 \\
Fair & 73 & 34.3 & 140 & 65.7 & 213 \\
Good & 130 & 55.6 & 104 & 44.4 & 234 \\
Knowledge on HEP & & & & & \\
Not satisfactory & 41 & 21.4 & 151 & 78.6 & 192 \\
Satisfactory & 182 & 53.2 & 160 & 46.8 & 342 \\
Distance to health Post & & & & & \\
Less than 5km & 145 & 45.2 & 176 & 54.8 & 321 \\
More than 5km & 79 & 36.6 & 135 & 63.4 & 213 \\
Service year of HEWS & & & & & \\
Less than 4 year & 188 & 42.5 & 254 & 57.5 & 442 \\
More than 4yaer & 35 & 38.0 & 57 & 62.0 & 92 \\
No. times home visited & & & & & \\
Not visited & 73 & 26.4 & 203 & 73.6 & 276 \\
$\begin{array}{l}\text { Once } \\
\text { Twice }\end{array}$ & 77 & 53.1 & 68 & 46.9 & 145 \\
Three times & 54 & 65.1 & 29 & 34.9 & 83 \\
\hline & 19 & 63.3 & 11 & 36.7 & 30 \\
\hline
\end{tabular}

\subsection{Determinants of HEP Utilizations: A Multilevel Logistic Regression Analysis}

A two-level multilevel analysis was used, kebeles as the second-level units and women as the first level units. Heterogeneity of HEP utilization among women across ten kebeles of the Dera district assessed by using chi-square test and showed that significant (Pearson chi-square value = 32.763 , df $=9, \mathrm{P}=0.001)$. Thus, there is an evidence for heterogeneity with respect to utilization of HEP across kebeles and multilevel logistic regression is attempted.

\subsubsection{Comparison of Multilevel Logistic Regression Models}

The deviance difference test and DIC of the models showed that the random intercept model was the better model 
as it had significant difference, and the smallest DIC value (531.46) compared to the empty (711.68) and random coefficient (537.07) models (Table 4). Hence, the random intercept model is a better model to fit HEP utilization.

Table 4. Comparison between multilevel logistic regression models.

\begin{tabular}{llll}
\hline & Deviance & Difference in deviance & DIC \\
\hline Empty model & 704.20 & \multirow{2}{*}{$195.38^{* *}$} & 711.68 \\
Random intercept model & 508.82 & 3.15 & 531.46 \\
Random coefficient model & 505.67 & & 537.07 \\
\hline
\end{tabular}

(**, significant at $1 \%$ level of significance)

\subsubsection{Results of Multilevel Empty Logistic Regression Model}

On average, not utilizing HEP in all kebeles included in the study is $57.3 \%$ [exp $(-0.2928) / 1+\exp (-0.2928) /]$ and approximately the same with the descriptive result (58.2\%). The variance of the random factor is 0.450 and significant at the $5 \%$ level of significance, and implies that there is significant variation in health extension service utilization among kebeles of the district with intra-kebele correlation $\mathrm{ICC}=12.06 \%$ as shown in Table 5 .

Table 5. Result of multilevel empty binary logistic model.

\begin{tabular}{|c|c|c|c|c|}
\hline Utilization of HEP & $\beta$ & S. $\mathbf{E}$ & $\mathbf{Z}$ & P-values \\
\hline Fixed part & & & & \\
\hline$\beta_{\mathrm{o}}($ constant $)$ & 0.2928 & 0.173 & 4.590 & $0.000 * *$ \\
\hline Random part & & & & \\
\hline$\sigma_{\mu_{0}}^{2}($ Cons $/$ cons $)$ & 0.450 & 0.149 & 3.02 & $0.0025 * *$ \\
\hline $\mathrm{DIC}=711.68$ & \multicolumn{4}{|c|}{$\mathrm{ICC}=0.1206$} \\
\hline
\end{tabular}

$(* *, 1 \%$ significance level)

\subsubsection{Results of Multilevel Random Intercept Logistic Regression Model}

The multilevel logistic regression model in Table 6 revealed Health Extension Service Utilization varied among kebeles of the district. The variance of the random term (kebele) is 0.183 with p-value 0.037 , which is significant at the $5 \%$ level of significance. Between-kebele level variance decreased from 0.450 in empty to 0.183 random intercept models. The reduction of the random intercept variance is due to the inclusion of fixed explanatory variables. Hence, some of the variation in HEP utilization, between Kebeles explained by differences in their fixed predictor variables. Additionally, the intra-region correlation was $5.27 \%$, implying that the percentage of variance of HEP Utilization could be attributable to the differences between kebeles when the fixed predictors are included

The fixed part revealed that being model in HEP, mother's education level, mother's employment status, mother's age, knowledge on HEP, attitude of HEWs, number of student and frequency of home visited by HEWs were found significant factors of HEP utilization in all kebeles at the 5\% level of significance. Whereas distances to health center and mean service year of HEWs were not significantly affect HEP utilization in the district and the effects of these factors were interpreted in terms of odds ratio as below.

Being a model in HEP was found significantly determine
HEP utilization at the $5 \%$ level of significance and the odds of households who were not model family in HEP was 3.27 (OR: $3.27 \mathrm{CI}:(1.78,6.01))$ times more likely than those who were model family in not utilizing HEP holding all other factors constant.

Education level of mothers (no educated and primary education) was found significant determinants of HEP utilization at 5\% level of significance and the odds households having mother's education levels of not educated was, 6.28 (OR: 6.28, CI: $(1.85,21.26))$ times more likely than those who had secondary and above education level in not utilizing HEP, keeping all other factors constant.

The employment statuses of mothers (farmer and daily labor) were significantly affected HEP utilization at 5\% level of significance. The odds of household not utilizing HEP based on employment status of mothers for those who were farmer and daily labor were, 2.00 (OR: 2.08, CI: $(0.72,1.95)$ ) and 4.41 (OR:4.41, CI: $(1.53,12.69))$ time more likely than those whose jobs were housewife, respectively, holding all other factors constant. While the odds of not utilizing HEP for Traders were statistically not significant at the 5\% level of significance. This indicates that utilizing HEP was for those women who were Trader and housewife were similar.

Similarly, mother's age group (25-34) was found significantly determine of HEP utilization at 5\% level of significance and the odds of mothers in the age group 25-34 was 1.53 (OR: $1.53, \mathrm{CI}(0.99,2.77)$ ) time more likely than those mothers in the age group 35-49 in not utilizing HEP, keeping all other factors constant. While the odd of mothers in the age group 15-24 was not significant, that means utilizing HEP was the same for those mothers in the age group 15-24 and above 35.

Knowledge on HEP was found significant determinants of HEP utilization at 5\% level of significance and the odds of not utilizing HEP for those households who had unsatisfactory knowledge on HEP was 3.26 (OR: 3.26, CI: $(1.95,5.44))$ times more likely than those who had satisfactory knowledge, fixing all other factors constant.

Number of students in the family also found to significantly affect HEP utilization at 5\% level of significance and the odds of not utilizing HEP was 0.75 (OR: 0.75 , CI $(.58, .97))$ times less as the number of student increase by one, keeping other factors constant.

Perceptions about attitude/conduct/ of HEWs (bad, fair attitude) were found to significantly affect HEP utilization at $5 \%$ level of significance. The odds of households who said that the HEWs had bad and fair attitude (conduct) were 3.34 (OR: 3.34, CI: $(1.68,6.64)$ ) and 1.967 (OR: 1.96, CI: (1.11, 3.48)) times more likely than those who had a good attitude in not utilizing HEP, respectively, fixing all other factors constant.

Finally, the number home visited by the HEWs found to significantly affect HEP utilization at 5\% level of significance and the odds of not utilizing HEP was 0.69 (OR: 0.69 , CI $(0.46, .79))$ times less as the number of time homes visited increase by one, keeping other factors constant. 
Table 6. Results of random intercept multilevel logistic regression model.

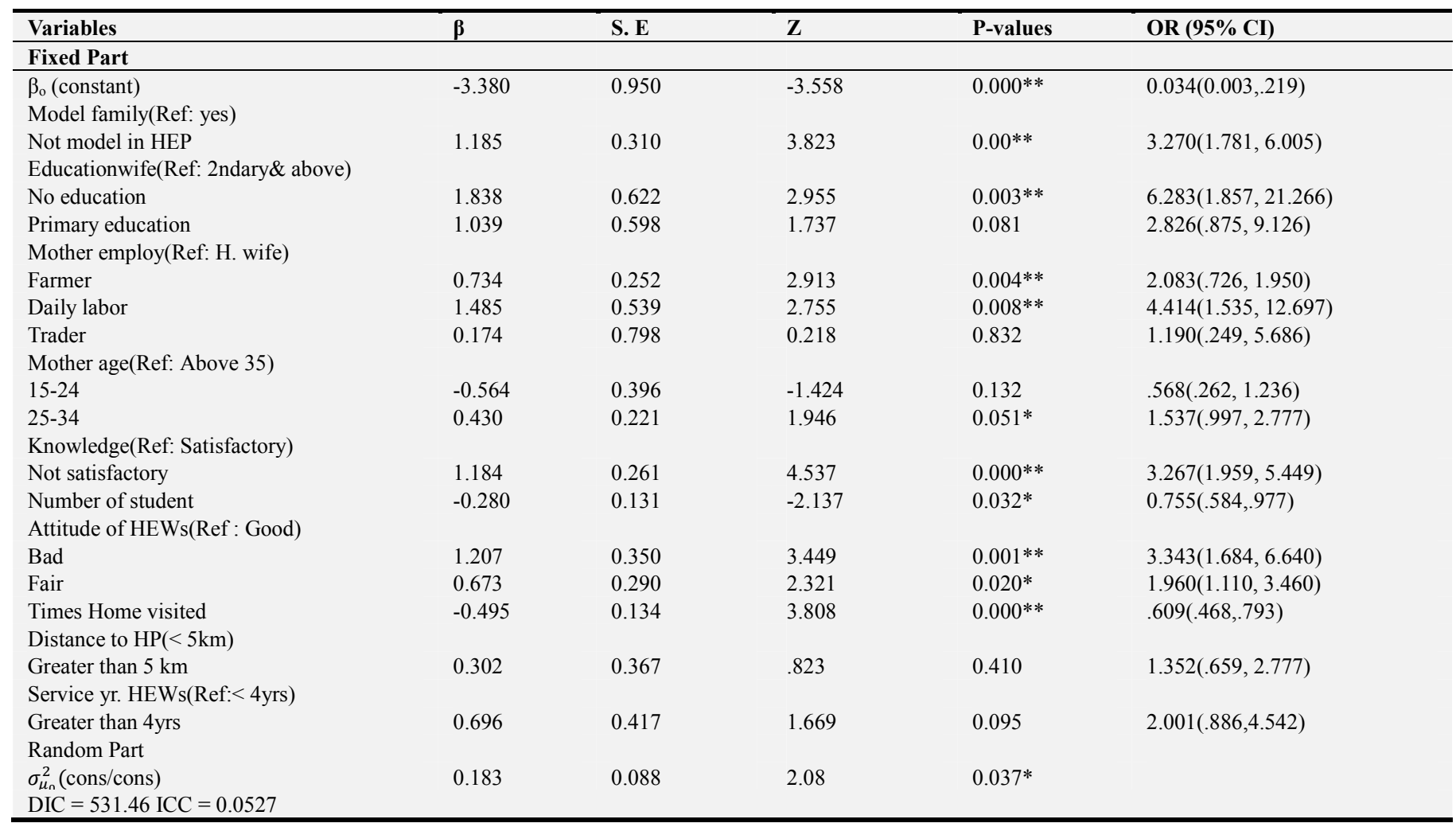

$\left(*,{ }^{*}\right.$ significant at $5 \%, 1 \%$ significance, CI: confidence Interval and Ref: is reference category)

\subsection{Discussion for HEP Utilization}

The random intercept revealed that factors such as the mother's age, mother's educational level, mother's employment status, knowledge on HEP, being model in HEP implementation, frequency of home visited by HEWs, Number of students in the family and attitude about of HEWs had a significant effect on health extension service utilization at $5 \%$ significance level and discussed.

The result revealed that being model in HEP had a significant effect on HEP utilization. The household whose family was model in implementing HEP expected to utilize more likely than those whose family was not model in HEP. The result also revealed that being model in implementing HEP had a highly significant effect with utilizing of HEP. The households who were not model in HEP had higher odds of not utilizing HEP than those who were model, which is in line with the previous studies done by $[16,17,19]$ that a model family utilized HEP more likely than non-model family.

Mothers' educational level is an important predictor of HEP utilization, in which HEP Utilization increase as mothers' education level increase. Studies showed that the lower maternal education level, the higher in not utilizing HEP. Educated mothers expected to have higher awareness and understanding about HEP than those who had no education. In this finding, the result showed that as the education level of mothers' increase, the odds of not utilizing HEP decrease, similar findings were obtained by $[15,16]$ as the education level of mothers increase, the odds of not utilizing HEP decrease.

Another determinant of HEP utilization is mothers' employment status. The household whose mother's employment status was a farmer was less likely to utilize HEP than those whose employment status was daily labor and others. In this finding, those households whose mother's employment status was house wife and Traders were less likely to not utilize HEP than those who were farmer and daily labor. This is consistent with the previous finding of [11] that mothers who were farmer and daily labor were less likely to utilize HEP than those who were merchant (Trader) and government employee.

The result revealed that mother's age had a significant effect with utilization of HEP. As mother's ages become older the proportion of not utilizing HEP increase. This is may be younger mothers have better understanding about the benefits of HEP than those who were older mothers. In this finding, the odds of not utilizing HEP for those mothers who were aged 25-34 was higher relative to those who were aged above 35 years in not utilizing HEP, similar finding was obtained by [11] that mother's age significantly affect HEP utilization.

Knowledge of the community on HEP was another factor that affects community utilization of HEP. The household who had satisfactory knowledge on HEP were more likely to utilize HEP than those who had unsatisfactory knowledge because the household who had satisfactory knowledge had 
more awareness about the importance of HEP than others. In this study, the household who had satisfactory knowledge, utilize more likely than those do who had unsatisfactory knowledge, similar findings were obtained by $[11,17,18,19]$ that those who had satisfactory knowledge utilize HEP more likely than those who had not satisfactory knowledge. However, it contradicts with [16] finding done on utilization of urban health extension program at Debretabor Town.

Perception of $\mathrm{HH}$ about the attitude (conduct of) HEWs had a significant effect with utilization of HEP. The household who had the perception about HEWs as bad and fair attitude toward the community were more likely to not utilize HEP than those who said HEWs had a good attitude toward them. In this finding, the household who said that HWEs had poor and medium attitude were more likely to not utilize HEP than those who said HEWs had a good attitude (conduct), similar finding was obtained by [17, 19] that attitude and HEP utilization had a positive effect.

Frequency of home visited by HEWs is another predictor of HEP utilization. The household those whose home visited frequently by the health extension workers were more likely to utilize HEP than those whose home was not visited by health extension workers. In this study, the household whose home was less frequently visited by health extension workers were more likely to not utilize HEP than those whose home visited, similar finding was obtained by $[17,19]$ that frequent home visit by HEWs increase HEP utilization.

\section{Conclusion and Recommendations}

\subsection{Conclusion}

The purpose of this study was to investigate the utilization of HEP determinants and variation among kebeles of Dera district, Oromia, Ethiopia based on a sample of 534 households. The study used multilevel binary logistic regression model to fit utilization of HEP.

The descriptive result revealed that out of 534 respondents, $223(41.8 \%)$ of them utilized and $311(58.2 \%)$ of them did not utilize health extension service given at the health post and outreach level.

Multilevel analysis is better to model the utilization of health extension program as to differentiate the variation among kebeles of the Dera district in HEP utilization. The result of models comparison indicated that the random intercept binary logistic regression model best fits the data for HEP utilization.

The results of random intercept binary logistic analysis identified that mother's age, mother's education level, mother's employment status, knowledge on HEP, being model in HEP, frequency of home visited by HEWs, number of students in a family, and perception about conduct of HEWs were significant determinants of HEP utilization.

\subsection{Recommendations}

Based on the finding of the study the following recommendation was forwarded.

i. The district health officer and HEWs should work on the perception of the community in order to enhance community's Health extension service utilization.

ii. The district health office should create-intensified awareness creation targeted to increase community understanding of Health extension service in order to increase utilization of health extension program of the community.

iii. Education level of mothers plays an important role in utilizing HEP. Thus, HEWs needs to focus on sustaining women with no education on HEP implemented to raise the utilization of HEP.

iv. The district health office should create an intensified training on model family to increase HEP utilization in the community.

v. Utilization of HEP varies among kebeles of the district significantly. Thus, the district health office has to work to reduce the variance and focus on the risk factors of utilization of HEP for each kebeles separately.

vi. A multilevel model is better to investigate effects of different determinants on HEP utilization and account variation across groups (clusters).

\section{Abbreviations}

DIC: Deviance information criteria, HEP: Health Extension Program, HC: Heath center, HEW: Health Extension Worker, HH: Household, PHCU: Primary Health Care Unit, ICC: Intra-class correlation.

\section{Availability of Data and Materials}

The dataset used and/ or analyzed during the current study are available from the corresponding author on reasonable request.

\section{Conflict of Interest}

The authors declare that they have no conflict of interests.

\section{Authors' Contributions}

AT was involved in the conception and design of the study, data collection and entry, interpretation and drafting the manuscript. DT and NA were involved in the design and review of protocol development, interpretation of the data and review of the manuscript. All authors read and approved the final manuscript.

\section{References}

[1] Federal Ministry of Health (FMOH) 2007. "Health Extension Program in Ethiopia - Profile.” Addis Ababa.

[2] World Bank. 2013. UNICO Studies Series 10, The Health Extension program in Ethiopia. Washington DC. 
[3] Assefa A, Alebachew H, Fassil N; Haniko M and Ihalainen A, B K. 2010. HIV/AIDS and the health related millennium development goals. The Experience in Ethiopia 2010, 369(2112): 2112-2311.

[4] Ghebreyesus A 2010. Achieving the Health MDGs: country ownership in four steps. 376(9747): 11228. (http://www.lancet.com/journals/lancet/article/PIIS0140 6736(10)61465- 1/full text)

[5] Federal Ministry of Health (FMOH) and Abt Associates. 2010. Fourth National Health Account Report. Addis Ababa.

[6] Dera district administrative: Health profile 2016.

[7] Central Statistical Agency (CSA) and ICF International. 2012. "Ethiopia Demographic and Health Survey 2011." Addis Ababa and Calverton, Maryland, USA: Central Statistical Agency and ICF International.

[8] CSA (Ethiopia), 2013. Population Projection of Ethiopia for All Regions at Wereda Level from 2014 - 2017: Addis Ababa, Ethiopia.

[9] Snijders, T. and Bosker, R. 1999. Multilevel Analysis: an Introduction to Basic and Advanced Multilevel Modeling. London. Thousand Oaks, New Delhi. Sage Publications.

[10] Peter C Austin. 2010. Estimating Multilevel Logistic Regression Models When the Number of Clusters is Low: A Comparison of Different Statistical Software Procedures. Int $J$ Biostat; 6(1): doi: 10.2202/1557-4679.1195.

[11] Kelbessa Zewudu, Baraki Negga and Egata Gudina. 2014. Level of health extension service utilization and associated factors among community in Abuna Gindeberet District, West Shoa Zone, Oromia Regional State, Ethiopia. BMC Health Services Research 14: 324. doi: 10. 1186/1472-6963-14-324.

[12] Anthony G. Turner. 2003. Sampling strategies. Review the Draft Handbook on Designing of Household Sample Surveys. ESA/STAT/AC.93/2.
[13] Gelman, A. and Hill, J. 2006. Data Analysis Using Regression and Multilevel or Hierarchical Models. Columbia University, Journal of the American Statistical Association.

[14] Goldstein, H. and Rasbash, J. 1996. Improved approximations for multilevel models with binary responses. J. Roy. Statist. Soc. A, 159: 505-513.

[15] Araya Medhanyie, Mark Spigt, Yohannes Kifle, Nikki Schaay, David Sanders, Roman Blanco, Dinant GeertJan and Yemane Berhane. 2012. The role of health extension workers in improving utilization of maternal health services in rural areas in Ethiopia: a cross sectional study. BMC Health Services Research 12: 352 doi: 10.1186/1472-6963-12-352.

[16] Yilkal Tafere, Mirkuzie Woldie, Henok Assefa, Amanu Aragaw 2014. Utilization of Environmental Health Services of Urban Health Extension Program and Associated Factors in Debretabor Town, North West Ethiopia: Cross Sectional Study. Science Journal of Public Health. Vol. 2, No. 5, 2014, pp. 494-501. doi: 10.11648/jsjph.20140205.28.

[17] Yitayal Mezgebu, Berhane Yemane, Worku Alemayehu and Kebede Yigzaw. 2014. The community-based Health Extension Program significantly improved contraceptive utilization in West Gojjam Zone, Ethiopia. Journal of Multidisciplinary Healthcare 2014: 7.

[18] Abajobir Alemu Amanuel and Seme Assefa. 2014. Reproductive health knowledge and services utilization among rural adolescents in east Gojjam zone, Ethiopia: a community-based cross-sectional study. BMC Health Services Research 2014, 14: 138. doi: 10.1186/1472-6963-14-138.

[19] Yitayal Mezgebu, Berhane Yemane, Worku Alemayehu and Kebede Yigzaw. 20014. Health extension program factors, frequency of household visits and being model households, improved utilization of basic health services in Ethiopia. BMC Health Services Research 2014, 14: 156. doi: 10. 1186/14726963-14-156. 